\title{
El debate entre transnacionalismo y nacionalismo metodológico como marco teórico para la comprensión del papel del empleo en la gobernabilidad de la inmigración en España
}

\author{
Álvaro Morcillo Espina \\ Universidad de Navarra. Centro Navarro de Desarrollo Internacional \\ almoes@gmail.com
}

\section{Resumen}

El debate entre el nacionalismo metodológico y el transnacionalismo ofrece múltiples oportunidades teóricas para los estudios migratorios. En este trabajo, se exponen las aportaciones que este debate brinda para la comprensión de la gobernabilidad de la inmigración en el sistema de estado autonómico español, partiendo del análisis de la gobernabilidad de la inmigración a nivel estatal, pasando por el nivel regional y acabando en el nivel local (provincial). Para ello, nos centramos en analizar el papel que el empleo desempeña en la gobernabilidad de la inmigración, destacando su relevancia y su significado en los tres pilares imprescindibles de cualquier política migratoria: el control, la integración y la cooperación al desarrollo. A nivel estatal, se analiza la legislación de extranjería y, a nivel regional, los planes de inmigración de las comunidades autónomas. En esos dos niveles, se sigue una metodología cualitativa (teoría fundamentada), mientras que, en el nivel local (provincial), se analiza y se comparan los resultados de la explotación de la EPA del primer trimestre de 2008 y del primer trimestre de 2009.

Palabras clave: teoría social; política de inmigración; integración social; cooperación al desarrollo; gobernabilidad; ciudadanía; estado nacional; globalización; comunidades autónomas.

Abstract. The debate between transnationalism and methodological nationalism as a theoretical frame to understand the role of employment for immigration management in Spain

The debate between methodological nationalism and transnationalism offers many theoretical opportunities for migration studies. In this paper, we describe the contributions that this debate provides for understanding the governance of migration within the Spanish autonomous state system. We analyse the governance of migration at the state level, the regional level, and the local (provincial) level. We focus on the role of employment in the governance of migration, highlighting its meaning and its relevance for the three essential pillars of migration policy: control, integration, and development cooperation. Using qualitative methodology (grounded theory) we discuss immigration-foreigner laws at the state level, and social integration plans at the regional level. At the local or provincial level 
we conduct a quantitative analysis on the basis of official statistic resources (Labor Force Survey).

Key words: social theory; immigration policy; social integration; cooperation for development; governance; citizenship; nation-state; globalization; autonomous communities.

\section{Sumario}

1. Introducción 5. El nacionalismo metodológico

2. Objetivo: el papel del empleo en la gobernabilidad de la inmigración

3. Metodología

4. La perspectiva transnacional

6. El debate entre transnacionalismo y nacionalismo metodológico para el caso español

7. Conclusiones

Referencias bibliográficas

\section{Introducción}

En España, los procesos migratorios de la última década han transformado la realidad social y, con el fin de conocerla mejor y tener datos fiables para la gestión de la inmigración, se han incentivado multitud de estudios en el mundo académico, en centros especializados y desde las propias administraciones públicas. Desde cada área y centro o grupo de investigación, se han escogido para analizar unas líneas preferentes del campo migratorio, conscientes de que abarcar todas las posibilidades y los interrogantes de este hecho no está al alcance de nadie. En el ámbito de nuestro trabajo (políticas públicas, mercado laboral, gobernabilidad, ciudadanía), son destacables numerosos estudios (Aja et al., 2006; Aláez Corral, 2006; Aparicio et al., 2002; Bonino et al., 2004; Iglesias de Ussel et al., 2010; Lucas y Díez Bueso, 2006; Solé et al., 2005; Zapata-Barrero, 2009b; Zapata-Barrero y Pinyol, 2008), que, sin embargo, aún no han conseguido, o no lo han intentado, construir un marco general de interpretación de la cuestión migratoria desde el punto de vista de la gobernabilidad de la inmigración dentro del complejo y enmarañado sistema autonómico del Estado español. Hay indicios, en determinados niveles de análisis, de las características que debería reunir ese marco teórico, pero hasta ahora nadie ha propuesto un modelo interpretativo que abarque a todos los órganos e instrumentos competentes en materia migratoria.

Indicios que se observan de que ese marco teórico está a punto de aflorar a la luz son, por ejemplo, el aumento de estudios en torno a la ciudadanía. En base a la perspectiva transnacional, a la existencia de actividades transnacionales que traspasan las fronteras, se ha extendido a nuestro país el debate sobre nuevos modelos de ciudadanía que no se basen en el estado nación (Aláez Corral, 2006; Lucas Martín, 2008; Zapata-Barrero y Requejo, 2001). La inmigración ha provocado, igualmente, una revisión crítica del modelo autonómico, del cual se han analizado las ventajas y los inconvenientes, los 
problemas de coordinación, la necesidad de descentralización para la correcta gestión de la inmigración y para atender a los inmigrantes desde un nivel próximo a ellos, como el nivel local. Por otra parte, la inmigración ha generado tensiones y conflictos entre los diferentes niveles de la Administración; los discursos de las fuerzas políticas se han etiquetado como demagógicos y poco realistas en muchas ocasiones (Pérez-Díaz et al., 2001); a nivel nacional y de la UE, se han hallado incompatibilidades entre los discursos de un mismo grupo político, lo cual ha generado confusión y duda sobre el proyecto migratorio de los gobernantes (Hix y Noury, 2007), y así un sinfín de observaciones que convendría ordenar para saber a qué atenernos al analizar la gobernabilidad de la inmigración en España.

En este trabajo, proponemos un marco teórico que se ajusta en muchos aspectos a la realidad migratoria nacional y a nuestro modelo de estado, lo cual facilitará la comprensión del rol que cada actor, especialmente la Administración pública, desempeña en la gobernabilidad de la inmigración desde una perspectiva sociológica y utilizando el «empleo» como eje articulador. En concreto, proponemos como un plausible marco teórico el debate conocido como transnacionalismo y nacionalismo metodológico.

Para quienes desconocen el origen de este debate, hay que decir que el transnacionalismo es la nueva perspectiva desde la que se están enfocando un gran número de investigaciones en el campo de las migraciones. El nacionalismo metodológico, en cambio, es definido como una versión clásica de observación de la realidad que presupone al estado nación como principio organizador de la sociedad y que, en el ámbito de las migraciones, también ha ejercido una gran influencia. Hoy, muchos especialistas se han decantado por la perspectiva transnacional como la mejor manera de explicar y describir los actuales procesos migratorios, que han adquirido una gran complejidad en el contexto de la globalización. La perspectiva transnacional, digamos que les ha proporcionado la flexibilidad y la agilidad que le faltaba a esa otra visión enraizada en la concepción del estado nación como marco omnicomprensivo del que resultaba difícil escapar. A pesar del éxito de la perspectiva transnacional en el mundo académico, todavía sigue habiendo muchas objeciones a esta perspectiva, ya que, si en lo académico está resultando de gran utilidad, en el plano político y social, todavía son predominantes las formas y las estructuras, las instituciones y los organismos, las normas y las costumbres que tienen su origen y su fundamento en la realidad del estado nación. Por este motivo, sigue habiendo investigadores que frenan, relativizan, piden prudencia a quienes consideran el transnacionalismo como la solución teórica definitiva para explicar los fenómenos migratorios del siglo XXI. En nuestro caso, conviene advertir al lector que nosotros no nos decantamos por ninguna de las dos perspectivas de forma exclusiva, sino que consideramos las ventajas y los inconvenientes que ambas ofrecen al investigador, y justamente en la combinación de ambas perspectivas es donde encontramos la mayor riqueza interpretativa para nuestro objeto de estudio, que no es otro que profundizar en el papel del empleo en la gobernabilidad de la inmigración para el caso de España. 


\section{Objetivo: el papel del empleo en la gobernabilidad de la inmigración}

\subsection{El papel del empleo...}

¿Qué quiere decir «el papel del empleo»? Desde una perspectiva sociológica, hay elementos de la vida cotidiana que desempeñan un papel importante en la sociedad. Pero no sólo elementos de la vida cotidiana, sino a veces también estructuras o instituciones que, poco a poco, adquieren un carácter funcional, con una finalidad conocida o desconocida, manifiesta o explícita, formal o informal (Merton, 1968; Parsons, 1999). El empleo, la posibilidad de trabajar, el poder estar ocupado en una serie de tareas a cambio de una remuneración, de un salario con el que tener la oportunidad de vivir y construir un proyecto vital, de futuro, aunque a veces las condiciones de vida no sean las óptimas, todo esto tiene, a nivel del individuo, unas connotaciones que no deben ser obligatoriamente las mismas que las que tiene el empleo a nivel de las instituciones o, en concreto, a nivel de las administraciones públicas y de los gobiernos, tanto de la nación, como de las regiones, como de los municipios.

Estos significados diferentes que el empleo puede adquirir aumentan cuando introducimos una nueva variable: los inmigrantes. Empleo e inmigración se han convertido en un binomio, no ahora, sino desde las primeras migraciones que se dieron en la Tierra, de seres humanos que se desplazaron en busca de mayores y mejores recursos, en busca de nuevos «trabajos» ${ }^{1}$ que posibilitaran mejorar las condiciones de vida de una tribu, de una familia, de una sociedad, de una nación o de un pueblo.

\section{2. ...en la gobernabilidad de la inmigración}

La gobernabilidad de la inmigración es la gestión de la inmigración. La inmigración ya no es definida como un problema a resolver. La inmigración es un hecho que hay que gestionar, como cualquier otro hecho o fenómeno social. ¿A quién le corresponde gobernar, gestionar la inmigración? A los gobernantes. Pero es ahí donde encontramos un contexto complejo para el caso de España. ¿Qué gobernantes? ¿Los que gobiernan a nivel estatal, regional o local? ¿O incluso los que están por encima del estado, en las instituciones supranacionales, en concreto en la Unión Europea? Es en este entramado de competencias locales, regionales, estatales y supranacionales donde el empleo desempeña un papel, tiene un rol desde el punto de vista de las políticas públicas, ejerce un papel en el nivel institucional.

Descubrir o interpretar el papel del empleo en un período de crisis económica nos parece que es algo imprescindible para conocer las partes y los elementos de este rompecabezas. El período en el que trascurre esta investigación abarca cuatro años, desde 2006 hasta 2010. En los dos primeros años

1. Entiéndase que las primeras migraciones no buscaban «trabajos», «empleos» como los concebimos hoy día, sino nuevas tierras para cultivar, zonas para pastorear, etc. 
(2006-2007), no se podía hablar de crisis económica, era, por esas fechas, un hecho impredecible, no existía crisis. En cambio, a partir de finales de 2007 y ya en 2008, la burbuja de crecimiento económico estalló, lo cual afectó a todo el planeta y, en el caso de España, las consecuencias han sido terribles, especialmente por la destrucción de empleo generada por la caída del sector de la construcción, que había sido el motor de desarrollo principal económico de los últimos años.

En todos esos años, la inmigración ha contribuido a cubrir las necesidades expansivas del mercado español, puesto que ha proporcionado numerosa mano de obra para los sectores con mayor crecimiento y demanda de trabajadores. Ahora, en un momento de destrucción de empleo, con más de 4 millones de parados, la población española se halla en una situación de gran incertidumbre sobre el futuro del mercado laboral (CIS) ${ }^{2}$, y la población inmigrante comparte esa incertidumbre, a la cual se añade la preocupación por la posibilidad de permanecer o no en el país (Arango et al., 2008).

En todo este período (y previamente también), se han producido cambios en la gobernabilidad de la inmigración que son más claros y evidentes a partir de la crisis económica. En cuanto a la complejidad de nuestro modelo de estado autonómico, se ha intentado al menos definir las competencias que corresponden a cada nivel de gobierno y dotarlos de recursos para desempeñar sus cometidos. Como decíamos más arriba, en este entramado de competencias, el empleo tiene un papel en el ámbito de la gobernabilidad de la inmigración, y hemos indagado en ese papel haciendo uso de la metodología de trabajo que exponemos a continuación.

\section{Metodología}

A nivel del Estado ${ }^{3}$, hemos profundizado en las competencias y en los instrumentos con los que se cuenta para gestionar la inmigración. Competencias que vienen recogidas en la Constitución y que se concretan en forma de leyes, de leyes de extranjería principalmente. Hemos analizado la legislación de extranjería, la hemos interpretado desde una perspectiva sociológica y hemos buscado la posición del empleo en dicho entramado legislativo.

A nivel de las regiones, hemos seguido un proceso similar, pero adaptado a las características de dicho nivel. Hemos analizado las competencias de los gobiernos autonómicos en materia de inmigración partiendo de unos instrumentos concretos y novedosos con los que cuentan las regiones: los planes de integración de inmigrantes. Hemos analizado el papel del empleo en dichos planes y hemos obtenido conclusiones sobre el mismo.

2. Barómetro del CIS de marzo de 2010: http://www.cis.es/cis/opencms/-Archivos/Indicadores/ documentos_html/TresProblemas.html

3. El análisis de la legislación de extranjería y de los planes regionales de inmigración ha formado parte del proyecto Las politicas de integración social de los inmigrantes, financiado por la Fundación BBVA y que recientemente ha sido publicado (véase Iglesias de Ussel et al., 2010). 
A nivel local ${ }^{4}$, hemos realizado una explotación novedosa de la EPA, al descender al nivel provincial y desagregando los datos por variables como edad, sexo, actividad, ocupación y nivel de estudios, diferenciando entre nacionales y extranjeros de regiones de inmigración. Hemos comparado las provincias de diferentes regiones en función de diversas variables y hemos obtenido nuestras conclusiones sobre el papel del empleo a nivel provincial (local) y sobre las semejanzas y las diferencias provinciales.

El análisis de cada uno de los niveles de gobierno (estatal, autonómico y provincial-local) se ha realizado bajo el prisma del debate entre transnacionalismo y nacionalismo metodológico, y se ha encontrado una respuesta a cada nivel sobre el papel del empleo en la gobernabilidad de la inmigración. En las siguientes páginas, describimos en profundidad todo el marco teórico al que nos referimos: la perspectiva transnacional y el nacionalismo metodológico. Más adelante, analizamos las consecuencias del debate entre transnacionalismo y nacionalismo metodológico a nivel del Estado, de las regiones y de las instituciones locales para el caso de España, para terminar con las conclusiones del trabajo.

\section{La perspectiva transnacional}

Previa a la definición de lo que nosotros queremos decir con transnacionalismo o perspectiva transnacional, nos resulta oportuna la diferenciación que señalan algunos autores sobre los diferentes usos del término y sus formas derivadas. De acuerdo con la concreción que Ibáñez Angulo (2008) realiza a partir de la distinción de Beck (2008) entre globalización como proceso, globalidad como producto resultante de este proceso y globalismo como la ideología que rige el proceso, la autora distingue entre transnacionalismo, transnacionalización y transnacionalidad:

El transnacionalismo o la perspectiva transnacional podría definirse como la ideología o la lógica que facilita el desarrollo de relaciones y de procesos y como la perspectiva teórica que analiza estos procesos. La transnacionalización incluiría el conjunto de procesos y de prácticas que, de forma más o menos efectiva, transcienden estas fronteras y la transnacionalidad (aún por definir sus contenidos específicos) sería el producto resultante de estos procesos. (Ibáñez Angulo, 2008: 9-10)

En nuestro caso, nos interesa centrarnos principalmente en la lógica o la perspectiva teórica denominada «transnacionalismo» o "perspectiva transnacional», así como en el potencial que esta nueva manera de observar la realidad nos ofrece para analizar el objeto de estudio que hemos elegido.

4. El análisis del empleo en las principales provincias con inmigración de España se ha realizado a partir de la explotación de los ficheros de microdatos de la EPA del primer trimestre de 2008 y del primer trimestre de 2009. 
La fundación de la perspectiva transnacional se atribuye a los estudios pioneros de Schiller et al. (1992b). Sin embargo, los trabajos de estas autoras no han dado lugar a una versión definitiva de qué es la perspectiva transnacional, sino que, por el contrario, sus aportaciones han sido criticadas por otros académicos (Goldring et al., 2007; Kivisto, 2001; Portes et al., 1999), lo cual ha provocado un variado abanico de significados sobre lo que se entiende por perspectiva transnacional y ha alimentado el debate teórico en torno a este concepto. Tras estos primeros trabajos sobre transnacionalismo (Blanc et al., 1995; Schiller et al., 1992a, 1992b), hubo una explosión de estudios que hacían uso de la palabra transnacional de manera indiscriminada en muchas ocasiones, lo cual originó una debilitación del término y de su potencial analítico, ya que a cualquier cosa se le ponía la etiqueta de transnacional. Esto provocó numerosas reacciones críticas y de rechazo hacia ese abuso que se estaba produciendo, y más sobre un tema que todavía no había superado un debate interno que verificara su validez (Goldring et al., 2007; Kivisto, 2001; Portes et al., 1999; Portes, 1999, 2001; Vertovec, 1999).

El surgimiento de un paradigma teórico que pretende responder a la existencia de un nuevo fenómeno debería, en términos mertonianos, someterse a una serie de pruebas que demuestren la validez y la oportunidad del mismo (Portes et al., 1999). El primer paso sería la demostración de la existencia del fenómeno como un fenómeno que implica a una proporción significante de personas en un universo relevante; que los hechos observados tengan una perdurabilidad, una estabilidad y aguante en el tiempo y, por último, que no existan ya conceptos que puedan aprehender esa realidad para no caer en la redundancia con el nuevo término. En otro trabajo, Portes (2001: 182) divide en tres partes la argumentación y la crítica a la perspectiva transnacional. La primera parte hace alusión al problema de los números, donde se critica la rápida expansión de la perspectiva transnacional que proveía en ese momento de nuevas hipótesis y argumentos frente a los modelos predominantes, especialmente el asimilacionismo. Otra causa de mayor peso para esa rápida expansión fue la metodología que se usó en los primeros estudios, ya que se seguía el principio de verificación más que el de falsación. Por ello, se tendía a buscar ejemplos y descripciones que apoyaran el nuevo paradigma y dejaran a un lado los ejemplos y los casos que no permitieran defender el modelo. La existencia de actividades transnacionales queda demostrada más tarde a través de otros estudios que siguen metodologías diferentes a las primeras, más rigurosas y amplias, por lo que la primera cuestión sobre si existen unas actividades que se pueden denominar "transnacionales» tiene una respuesta afirmativa. Ahora bien, aún queda por esclarecer si dichas actividades transnacionales son diferentes a otras actividades semejantes que se han dado anteriormente en los grupos de inmigrantes. Algunos de los primeros autores que defendieron el transnacionalismo, al comprobar que, en tiempos pasados, se habían dado fenómenos semejantes, abdicaron en la defensa de esta perspectiva. Sin embargo, autores que inicialmente fueron más críticos, apoyados en investigaciones y revisiones más sólidas, confirmaron la existencia de algo nuevo y diferente 
(Schiller, 1999) que permitía hablar de actividades transnacionales (Goldring et al., 2007; Kivisto, 2001; Levitt, 2001; Portes, 1999, 2001; Vertovec, 1999).

Portes (2001: 184), apoyándose en Merton (1968), desbarata los obstáculos que se plantean a la novedad del transnacionalismo, argumentando que cualquier descubrimiento en ciencias sociales siempre va precedido de la existencia de indicios que hacen presagiar la existencia de algo nuevo, pero que el mérito está en presentar ese algo nuevo a la comunidad científica y conceptualizarlo correctamente. Entonces, con el nuevo hallazgo sobre la mesa, se puede decir que aquellos fenómenos pasados, aquellos indicios, también pueden definirse según este nuevo concepto, pero que si no se hizo anteriormente, es porque nadie había sido capaz de demostrar la relevancia ni la singularidad del mismo.

Un último obstáculo que el transnacionalismo debe superar es la multitud de significados que se atribuyen al término y la variedad de expresiones derivadas de él (Gowricharn, 2009; Kivisto, 2001; Portes, 2001; Vertovec, 1999). Cada autor o grupo de académicos se decanta por alguna de estas expresiones, que muchas veces son acuñadas por ellos mismos buscando añadir algún matiz del que carece la expresión usada por otro autor. El término campo social transnacional (Blanc et al., 1995; Schiller et al., 1992a) dio paso a los espacios transnacionales (Faist, 2000b), en los que las comunidades transnacionales (Vertovec, 1999) también tenían su lugar, algo que no sucedía en el transnacionalismo de Portes, Guarnizo y Landolt (1999), que reduce la unidad de análisis al individuo y a la red familiar. Como dice Gowricharn (2009: 3), las redes transnacionales y las cross-border activities son expresiones que se usan sin excesivo rigor o con cierta flexibilidad por parte de los estudiosos de las migraciones.

Portes (2001: 187), en un último intento de no dejar este asunto sin resolver, establece una tipología de «actividades fronterizas» en función de quien las dirige, distinguiendo los siguientes actores: los estados nacionales, las instituciones formales asentadas en un único país, las instituciones formales que existen y operan en varios países y los actores no institucionales que provienen de la sociedad civil. A partir de esta tipología de actores, clasifica las actividades y los programas de los estados nacionales y de las instituciones que tienen su sede en un único país como internacionales, pues existe una clara afiliación nacional de esos actores. Califica como actividades multinacionales a las que realizan instituciones formales que operan en varios países y, por tanto, sus intereses no están claramente ligados a un estado nación, sino que se expanden por multitud de lugares. Actividades transnacionales serían las que promueven actores no institucionales organizados en grupos o en redes de individuos a través de las fronteras, Muchas de dichas actividades son informales y quedan al margen del control y de la regulación de los estados (Portes, 2001).

Para comprender las aportaciones de la perspectiva transnacional a nuestro trabajo, es necesario presentar el debate entre transnacionalismo y nacionalismo metodológico como un juego de luz y sombra, en el que cada parte adquiere un sentido claro en oposición a la otra, de manera que puede decirse que del 
transnacionalismo, tal y como lo usamos aquí, sabemos mejor lo que no es que lo que es realmente. Esto es algo que no solo nos sucede a nosotros, sino que resulta como consecuencia de la variedad de significados que el término transnacional ha ido adquiriendo desde su origen. El intento de delimitación de diversos autores (Kivisto, 2001; Portes et al., 1999; Portes, 1999, 2001; Schiller et al., 1992a; Vertovec, 1999) ha conseguido frenar el uso abusivo del término, pero, al no llegar a un claro acuerdo ni siquiera entre ellos sobre lo que significa perspectiva transnacional o actividades transnacionales (Gowricharn, 2009), el resto de investigadores tiene la opción de seleccionar lo que más pueda interesarles de cada una de las definiciones, con lo cual elaboran su propio esquema conceptual y argumentan con el mayor rigor posible la elección hecha.

Así, los más recientes trabajos de Glick-Schiller (Schiller y Caglar, 2009; Schiller et al., 2006; Schiller y Caglar, 2007), basados en las posibilidades analíticas de la perspectiva transnacional en oposición a las limitaciones del nacionalismo metodológico (Wimmer y Schiller, 2002b, 2003), nos han proporcionado ideas muy sugerentes para nuestro análisis. Por ese motivo, no hemos seguido profundizando en la cuestión cultural del transnacionalismo, ni en el estudio de los inmigrantes a nivel individual o de sus redes familiares (Portes, 2001), ni en las comunidades transnacionales, ni en si el transnacionalismo es una forma de asimilación (Kivisto, 2001) o una alternativa (Guarnizo et al., 2003). Digamos que nuestro objeto de análisis está más próximo a las medidas que los estados y las administraciones elaboran en el ámbito de las migraciones, y que, según la clasificación de Portes (2001), entraría dentro de lo que se llaman «actividades internacionales en el área política». Pero dichos programas y actividades impulsados «desde arriba» (Smith y Guarnizo, 1998), aunque no puedan recibir el nombre propio de transnacionales, sus consecuencias sí pueden ser analizadas desde el debate que presentamos entre la perspectiva transnacional y el nacionalismo metodológico, ya que dichas acciones están en continua tensión con las actividades transnacionales que llevan a cabo «desde abajo» los grupos de inmigrantes y las organizaciones no institucionales y «desde arriba» los organismos supranacionales, grandes corporaciones globales (Ibáñez Angulo, 2008). Estas actividades transnacionales generan nuevas situaciones que se escapan a la regularización y al control del estado. Por tanto, también afectan al significado de la soberanía estatal y llevan a preguntarse por la perdurabilidad de los estados tal y como se han concebido en la tradición clásica y sobre la ciudadanía (Hammar, 1990; Soysal, 1994). Por último, para ordenar y posicionar a algunos de los autores mencionados, merece la pena prestar atención a la clasificación que Kivisto (2001) realiza a partir de la antropología, la sociología y la ciencia política.

Desde la antropología cultural, los trabajos de las autoras pioneras (Blanc et al., 1995; Schiller et al., 1992a, 1992b) parten de dos puntos iniciales en su definición del transnacionalismo: la cuestión histórica y la cuestión teórica. Desde la revisión histórica, destacan las diferencias cualitativas que se dan en los inmigrantes de hoy en comparación con los inmigrantes de finales del siglo xIx o principios del Xx. Principalmente, destacan que los inmigrantes de 
esa época anterior rompían las relaciones con su tierra, al mismo tiempo que los lazos culturales se desvanecían, con lo cual iniciaban una nueva andadura en solitario en la sociedad de acogida y se introducían en una nueva atmósfera o contexto cultural, social y económico. En cambio, los inmigrantes de hoy mantienen redes, actividades y modelos de vida que andan entre su sociedad de origen y su sociedad de acogida. Sus vidas trascienden y atraviesan los límites nacionales haciendo que dos sociedades diferentes confluyan en una única sociedad transnacional (Kivisto, 2001).

A partir de esta primera puntualización histórica, las autoras definen los términos transnacionalismo y transmigrante. El transnacionalismo es «el proceso por el cual los inmigrantes construyen campos sociales en los que quedan unidos tanto el país de origen como el de acogida", mientras que los transmigrantes serían los inmigrantes que construyen tales campos sociales sosteniendo una extensa variedad de relaciones sociales instrumentales y afectivas que traspasan las fronteras (Blanc et al., 1995; Schiller et al., 1992b). Kivisto (2001: 553) argumenta que una de las principales y más originales aportaciones de estas autoras es que las ciencias sociales no deberían estar limitadas en su unidad de análisis, ya que el problema principal de las teorías que operan como sistemas cerrados es que, al final, acaban reduciendo la unidad de análisis al estado nación y, por tanto, se encuentran con serias dificultades para responder a los interrogantes que los inmigrantes de hoy suscitan. Esta aportación ha tenido buena acogida entre los investigadores, ya que engarza en el extenso debate sobre el papel del estado nación (Sassen, 1996: 1-2) que tiene lugar en el mundo globalizado 5 .

En nuestro estudio, esta es una de las aportaciones principales que el transnacionalismo nos ofrece, ya que, en el caso de España, sigue siendo práctica común analizar la realidad social, también la inmigración, dentro de límites análogos a los creados por el estado nación. La parcelación y la limitación de la unidad de análisis por fronteras políticas convencionales, como las regiones o las provincias, no deja de ser un obstáculo más para el conocimiento profundo de las conexiones que los inmigrantes mantienen entre la sociedad de acogida y la sociedad de origen (Llopis Goig, 2007).

En el ámbito de la sociología, Kivisto (2001) declara a Portes como el investigador a través del cual se ha popularizado y se ha extendido más la perspectiva transnacional. Tomando los primeros trabajos de Portes en los que trata este tema, analiza su postura y las diferencias con Schiller y compañeras, y define el trabajo de Portes como un intento de elaborar una teoría de medio alcance según conceptos mertonianos. De Portes destaca positivamente la revisión histórica, profunda y rigurosa que hace para diferenciar a los inmigrantes de antes y a los de ahora, dando validez al uso de la nueva perspectiva transnacional,

5. En este debate se cuestiona el actual papel que el estado nación desempeña en las relaciones políticas, económicas y sociales que se producen en un mundo globalizado en el que las grandes multinacionales y los organismos internacionales parecen haber erosionado el tradicional poder soberano de los estados. 
pero sin que se puedan etiquetar todas las actividades de los inmigrantes de hoy como transnacionales. En su trabajo (Portes et al., 1999; Portes, 1999), rechaza la necesidad del término transmigrante, puesto que considera suficiente la palabra inmigrante para abarcar ese significado. Sin embargo, puntualiza que el uso del concepto transnacionalismo debe limitarse a aquellas actividades que implican continuidad de las relaciones sociales a través de las fronteras y en el tiempo. Para Portes (Kivisto, 2001: 560), la adecuada unidad de análisis serían los individuos y sus redes, con lo que excluye a las comunidades y a las estructuras superiores como los gobiernos. Para realizar esta exclusión, distingue entre un transnacionalismo "desde abajo» y un transnacionalismo "desde arriba». El primero es el que se puede aplicar a la inmigración, mientras que el segundo es el que corresponde a las corporaciones globales y a los gobiernos. La exclusión de las comunidades transnacionales es razonada en base a criterios metodológicos en los que la familia y los individuos serían la unidad de análisis realmente interesante y factible para estudiar.

En tercer lugar, desde el campo de la ciencia política, Kivisto señala el trabajo de Faist (2000a) como el más preciso y ambicioso desde el punto de vista conceptual, puesto que supera las carencias que, desde la antropología y la sociología, ofrecían los autores anteriores. Faist ofrece un marco teórico en el que el transnacionalismo estaría entre la teoría mundial de sistemas y la teoría de la globalización. La distinción con la primera está en la posibilidad de los inmigrantes de tener relaciones entre su país de origen, situado en la periferia, y más de un país de destino, situado en el núcleo o centro. Con respecto a la teoría de la globalización, la principal distinción estaría en cómo las dimensiones de espacio-tiempo se diluyen en la teoría de la globalización, al intensificarse las relaciones y reducirse las distancias en la comunicación gracias a los nuevos avances tecnológicos. La aportación de Faist que más interés nos suscita es el concepto de espacios sociales transnacionales, una idea que tanto Portes como Schiller tratan en sus trabajos, pero con menor claridad que Faist, cuando hablan del campo social.

By transnacional spaces we mean relatively stable, lasting and dense sets of ties beyond and across the borders of sovereign states. They consists of combinations of ties an their contents, positions in networks and organisations, and networks of organisations that cut across the borders of at least two nationstates. Transnational spaces differ from clearly demarcated state territories. Nonetheless, actors in transnational spaces may include state agents who operate on behalf of a national state government or an intergovernmental organisation. (Faist y Özveren, 2004: 4).

Además de incidir en la distinción entre transnacionalismo desde arriba y desde abajo, y de caracterizar cada uno de ellos con más exactitud que Portes, Faist también incluye en su marco teórico la posibilidad de estudiar a las comunidades transnacionales (Faist y Özveren, 2004: 9). De hecho, enfatiza que el papel de los estados es clave para determinar el porvenir de las comunidades transnacionales, especialmente por ostentar el poder para definir las 
condiciones o características de la ciudadanía (Kivisto, 2001: 572). Justamente nos quedamos con esta última idea de Faist para continuar más adelante exponiendo el papel de los estados en la definición de la ciudadanía desde el marco teórico del transnacionalismo.

El trabajo crítico de Kivisto (2001) es una buena aportación desde el punto de vista de la metateoría o de la epistemología de la perspectiva transnacional, pero no acaba de satisfacernos la excesiva crítica que realiza al trabajo antropológico de Schiller y compañeras, cuando, en concreto, Schiller ha seguido haciendo muy notables aportaciones al avance y a la maduración de esta perspectiva con sus trabajos posteriores (Schiller y Caglar, 2009; Schiller et al., 2006; Schiller y Caglar, 2007). Así mismo, nos parece que el trabajo de Portes se salva de otras críticas por la precisión de su metodología, por la cuantificación de sus resultados, por la concreción tan exhaustiva de su unidad de análisis. Por último, Faist presenta un trabajo más teórico, en el que hay una mayor profundidad argumentativa, pero donde, al mismo tiempo, se observa la carencia de contacto directo con la realidad de la inmigración.

De las tres corrientes o visiones, se puede extraer una idea común, que la perspectiva transnacional pretende facilitar la comprensión de la interconectividad de personas y lugares en todo el planeta, en un momento en el que las tecnologías de la comunicación y el desarrollo de los medios de transporte han modificado las pautas y los tiempos de los seres humanos (Beck, 2008; Kivisto, 2001; Portes et al., 1999; Schiller et al., 1992b). Las migraciones internacionales están en el centro de atención de estos procesos, ya que ponen en juego multitud de elementos que acaban influyendo en la configuración de cómo la gente y los lugares están conectados a través del espacio y del tiempo en su dimensión económica, política, social y cultural (Goldring et al., 2007; Portes et al., 2008b; Smith y Guarnizo, 1998). Así mismo, las migraciones internacionales constituyen una pieza clave porque nos proponen ejemplos constantes de personas, inmigrantes de hoy, que viven en un espacio transnacional, es decir, en un espacio social que no está propiamente ni en el lugar de origen ni en el lugar o lugares de destino, sino que es justamente el resultado de la combinación de ambos.

\section{El nacionalismo metodológico}

Se suele definir el nacionalismo metodológico como la suposición de que el estado nación es la forma natural y necesaria de la sociedad en la modernidad y, por lo tanto, el estado nación es considerado como el principio organizador de la modernidad (Chernilo, 2006; Wimmer y Schiller, 2002a, 2002b, 2003). Ahora bien, más que quedarse en una simple suposición, los críticos del nacionalismo metodológico proponen una revisión del papel del estado nación en la configuración de la sociedad y de las instituciones, de la ciencia y de la cultura, prácticamente de cualquier área, porque la tesis que defienden es que el estado nación como principio organizador ha penetrado y ha conformado todas las estructuras sociales. 
En torno a 1970 es cuando aparecen los primeros trabajos que llaman la atención sobre la estrecha unión conceptual de la «sociedad» y la formación histórica del estado nación. La crisis de la modernidad empieza a despertar la revisión crítica del estado nación, de la teoría sociológica, de la sociedad misma. La postmodernidad reclama nuevos paradigmas explicativos en un mundo globalizado en el que las estructuras parsonianas que habían guiado gran parte de la investigación social, no hallan respuesta para los nuevos fenómenos sociales, para las nuevas formas de relación, para una nueva experiencia del espacio y del tiempo.

Es Herminio Martins (1974) el primero en acuñar el término nacionalismo metodológico, al hacer una crítica al modo en el que, en los últimos años, se estaba llevando a cabo la investigación social por parte de muchos académicos, que utilizaban los conceptos derivados del estado nación, en concreto sus límites, para delimitar a la sociedad como principal objeto de estudio. Esta manera de concebir a la sociedad, identificándola con el estado nación, ha conducido a una homogeneización de toda la unidad de análisis, de toda la sociedad, como un ente cultural y socialmente uniforme (Wimmer y Schiller, 2002a). La consecuencia principal de aceptar las concepciones del estado nación en el estudio de la sociedad ha sido la identificación entre estado nación y sociedad como una misma unidad de análisis (Smith, 1979). En tal tipología de sistema, donde el estado nación o la sociedad están envueltos por el ambiente al que deben adaptarse para sobrevivir, el cambio social acaba siempre explicándose a raíz de factores internos del propio sistema. Una explicación que hoy, con una visión tan extendida de las consecuencias de la globalización, ha desaparecido por completo, lo cual ha provocado que casi cualquier persona sea consciente de que el cambio social en el siglo xxi puede tener su origen en factores externos producidos en cualquier lugar del planeta.

De acuerdo con Chernilo (2006), Martins resalta las consecuencias del nacionalismo metodológico en el desarrollo de las disciplinas, de la ciencia, mediante la creación de categorías sociológicas fundadas en el estado nación. En cambio, Smith se centra en cómo el mismo estado nación busca consolidarse a través de un sistema internacional de estados nación que refuerza el papel del estado nación a nivel social, intelectual y político. En la línea de Smith, Ibáñez (2008) menciona también la existencia de una lógica internacional o «internacionalidad» a raíz de la definición de Rée (1992), en la que la clave está en la creación de un sistema de organización en el que siempre pudiera exigirse una responsabilidad a una nación por los actos de cada ser humano y en cada lugar del planeta, es decir, que mediante la ciudadanía y mediante la soberanía sobre el territorio, todos los individuos quedaran adscritos a un estado nación y cada trozo del planeta correspondiera a algún estado.

El hecho de que surgieran las primeras críticas o revisiones del modelo analítico y metodológico que la sociología había utilizado en los últimos años, ya suponía una novedad y una entrada de aire fresco en el mundo académico. La simple revisión o reflexión sobre un modelo que se había instalado fuertemente en el mundo científico permitió profundizar en las consecuencias de 
ese modelo sobre los resultados que se habían obtenido y sobre la incapacidad para resolver preguntas que no encajaban en las limitaciones o en los encuadres de una sociedad identificada con un estado nación. El estado nación podía seguir siendo principio organizador de la sociedad de ese momento, pero ya no sería de forma inconsciente o velada, sino manifiesta. El nacimiento de las ciencias sociales en un contexto fuertemente influenciado por la creación de los estados nación, hizo que éstas tomaran la realidad del estado nación como un constructo indiscutible que acabó en una compartimentalización de la ciencia en áreas o disciplinas «nacionales», influenciada no sólo por el nacionalismo, sino también por las organizaciones que éste había creado, como las universidades, los departamentos o los centros de investigación (Wimmer y Schiller, 2002b, 2003).

Wimmer y Schiller (2002a, 2003), en su crítica a este paradigma, destacan tres vías por las que se llega a la aplicación del nacionalismo metodológico ${ }^{6}$ en el análisis de la realidad social:

a) La primera manera para situarse dentro del nacionalismo metodológico es ignorar, obviar o despreciar la importancia fundamental del nacionalismo para la configuración de las sociedades modernas.

b) Si este primer paso se combina con la naturalización del estado nación, se acaba en una posición en la que se da por supuesto que los límites del estado nación delimitan y definen la unidad de análisis de las investigaciones.

c) La tercera fase o vía para situarse en el nacionalismo metodológico es la limitación del estudio de los procesos sociales en función de las fronteras políticas o geográficas de un estado nación particular.

Identificado el eje sobre el cual se había construido gran parte del conocimiento del último siglo y viendo las dificultades que el mundo moderno tenía para responder a las preguntas más elementales, quedaba la opción de proponer un nuevo principio organizador de la sociedad y del mismo conocimiento que no fuera, por tanto, el estado nación. A raíz de esta crítica del estado nación como principio organizador de la modernidad, y entrando en una época calificada como de «crisis de la modernidad», «postmodernidad» o «modernidad tardía» entre las variadas denominaciones existentes, surgen otras alternativas al modelo de estado nación que buscan explicar certeramente la sociedad actual, como, por ejemplo, el cosmopolitanismo, el transnacionalismo o el globalismo (Beck, 2007, 2008).

Dentro del campo de las migraciones, el término más utilizado es el transnacionalismo, las migraciones transnacionales. No se suele hablar de migraciones globales o cosmopolitas, porque no se adaptan correctamente al fenómeno migratorio tal y como lo hace la perspectiva transnacional, ni al concepto de actividades transnacionales que suelen ser objeto de estudio. Por este motivo,

6. En concreto, se refieren a una consecuencia del nacionalismo metodológico que denominan «lente étnica». 
nos decidimos por la dualidad formada por nacionalismo metodológico frente a transnacionalismo, y no frente al cosmopolitanismo o globalismo. La lógica transnacional como «un estilo de pensamiento y una forma de estructurar las relaciones sociales que se desarrolla en un campo social transnacional cuyos límites no son coincidentes con los del estado nación» (Ibáñez Angulo, 2008) es nuestra alternativa al nacionalismo metodológico en el campo de las migraciones.

\section{El debate entre transnacionalismo y nacionalismo metodológico para el caso español}

\subsection{El nivel estatal}

El estudio del papel del «empleo trabajo» a nivel estatal, en el contexto de la gobernabilidad de la inmigración, se ha realizado mediante metodología cualitativa, siguiendo los principios de la teoría fundamentada (Glaser y Strauss, 1967) y teniendo la legislación de extranjería como objeto de estudio, ya que es el principal instrumento con el que cuenta el estado para ejercer sus competencias (Miret i Serra, 2009). La dificultad principal de este análisis es la inestabilidad de gran parte de la legislación de extranjería, tanto en Europa como en España. En la última década, los cambios y las reformas jurídicas que se han llevado a cabo en cuestiones de inmigración y extranjería han dado para escribir muchos libros y artículos sobre las motivaciones que hay detrás de esas reformas y las consecuencias que tienen en el conjunto de los estados miembros (Zapata-Barrero, 2009a, 2009b), en la soberanía de los mismos, en los modelos de ciudadanía, en el control de las fronteras, en el mercado de trabajo, en la garantía de principios humanitarios e incluso en el futuro de la Unión Europea.

Desde nuestro esquema teórico de nacionalismo metodológico y transnacionalismo, el análisis de la legislación de extranjería en contextos de multiculturalidad nos lleva directamente hacia el debate sobre los nuevos modelos de ciudadanía. El debate sobre los nuevos modelos de ciudadanía afecta no sólo al caso español, sino también al resto de países de la Unión Europea y a la Unión como ente supranacional que igualmente reclama la existencia de una ciudadanía europea. El estudio de la gestión de la inmigración a nivel estatal nos obliga a prestar especial atención a esta cuestión, ya que forma parte de las competencias del estado todo lo que se refiere a la concesión de la nacionalidad $\mathrm{y}$ al control de las fronteras 7 .

Zapata-Barrero $(2003,2005)$ detalla con gran precisión la relación existente entre estado, nación y ciudadanía. Esta tríada y el desarrollo de las relaciones entre cada una de las partes que la forman está inserta en el debate entre nacionalismo metodológico y transnacionalismo. La relación entre estado, nación y ciudadanía se establece de la siguiente manera cuando hablamos del contexto

7. Artículo 149.1 de la Constitución Española. 
europeo, ya que en el contexto americano el papel de la nación lo desempeña la Constitución:

Estado es la entidad política que define quién es ciudadano y qué limita territorialmente su actividad. La Nación es la entidad simbólica que vincula culturalmente el territorio estatal con la ciudadanía, creando la lealtad y cohesión necesarias para que el vínculo entre el Estado y la ciudadanía sea permanente y estable a través del tiempo. La Ciudadanía [...] es el principal vehículo que tienen el Estado y la Nación para vincularse y legitimarse. (Zapata-Barrero, 2003: 175)

Esta relación triádica requiere de los tres elementos para poder subsistir, de modo que no puede concebirse, dentro del ámbito europeo, un estado sin una definición de nación, ni tiene pleno sentido un estado y una nación sin ciudadanía, etc. (Zapata-Barrero, 2003). Esta tríada formada por estado, nación y ciudadanía (ENC) es una de las más claras consecuencias del «nacionalismo metodológico» (Gil Araújo, 2009; Wimmer y Schiller, 2002b, 2003) y está tan enraizada en Occidente que buscar una nueva manera de entender la realidad fuera de ese paradigma resulta harto complicado.

En los nuevos contextos de multiculturalidad y en un mundo globalizado en el que las fronteras pierden su tradicional función cuando se trata de la circulación de bienes, información e incluso de personas de diferentes nacionalidades, la tríada ENC no alcanza a explicar las nuevas relaciones y realidades emanadas de esos flujos. Sin embargo, cualquier intento de salir de este paradigma se encuentra con serios obstáculos, entre ellos el de la inseguridad y la duda que genera cualquier nuevo planteamiento (Zapata-Barrero, 2003).

Según Zapata-Barrero (2003), el multiculturalismo se situaría dentro de los principales debates que tienen su punto de mira en la relación de la tríada ENC, con la capacidad de acertar en la descripción de los puntos débiles que presenta y aportando soluciones que el autor denomina «líneas de razonamiento»:

a) Linea de razonamiento conservadora: el vínculo ENC tiene carácter indisoluble, no puede desaparecer y, por tanto, el multiculturalismo, que genera ciertos temores y dudas sobre la eficacia de esa tríada para explicar las nuevas realidades, conduce al reforzamiento de lo que se conoce y ha funcionado desde hace años, la relación ENC clásica.

b) Linea de razonamiento revolucionaria: «el multiculturalismo constituye una amenaza seria» a la tríada ENC, puesto que afecta a los paradigmas políticos.

Para este autor, el primer razonamiento estaría en la base de lo que él denomina «vía fundamentalista». Para Wimmer y Schiller (2003), sería una forma de concebir la realidad y, en concreto, esta tríada ENC estaría fuertemente enraizada en la "naturalización» del estado nación como algo sobre lo que no se puede discutir ni ponerse a prueba. Ambos autores destacan el proceso de 
homogeneización de la sociedad al que da lugar esta defensa de la tríada ENC como si de algo sagrado se tratara. Se concibe la sociedad como un grupo homogéneo, uniforme en términos culturales y se identifica la ciudadanía con la nacionalidad (Aláez Corral, 2006; Zapata-Barrero, 2003).

La otra vía que define Zapata-Barrero (2003) es la «vía multicultural», que «en contraste con la primera, [...] problematiza la conexión ENC». Lo que añade esta vía es el «carácter heterogéneo de la sociedad y también de la ciudadanía, que no debe estar identificada con una única nacionalidad». Este autor pone como ejemplo el caso de la ciudadanía europea, a la que se llega desde varias nacionalidades que se identifican con ciudadanías de estados miembros. También añadimos el caso de España, como ejemplo claro de la evolución del concepto de ciudadanía y del surgimiento de nuevos modelos ligados a las diferentes «naciones» que se reconocen recientemente en los estatutos autonómicos de varias regiones ${ }^{8}$ (Gil Araújo, 2009). Dentro de nuestro esquema teórico general, la vía multicultural se aproxima a la perspectiva transnacional más que al nacionalismo metodológico, que sería propio de la vía fundamentalista. La perspectiva transnacional trata de aportar soluciones similares a la vía multicultural, partiendo de una concepción de la realidad más compleja, nada homogénea, en la que hay movimientos y asentamientos de personas con diversas identidades culturales y nacionalidades. La apuesta por una vía multicultural o por una vía fundamentalista se refleja en las leyes que las instituciones aprueban.

En cuanto al papel del empleo en la gobernabilidad de la inmigración a nivel estatal, vemos ${ }^{9}$ que el código central sobre el que gira la legislación de extranjería es el control, que no es únicamente control de flujos, sino también control social (Iglesias de Ussel et al., 2010: 96), en forma de control administrativo-policial y control de flujos. En torno al control social, gravitan otras familias de términos, la más destacada de las cuales es la del «mercado de trabajo» o sencillamente "trabajo empleo». El control (social) que ejerce el estado tiene como uno de sus principales objetivos la protección de la ciudadanía, la regularización y el control de quién puede adquirir la ciudadanía, estableciendo las condiciones necesarias para alcanzarla. En el conjunto de requisitos y condiciones que el estado determina, el «trabajo» se convierte en un elemento indispensable de todo el proceso, ya que todos los pasos para obtener la nacionalidad se inician en la mayoría de los casos por la incorporación regularizada al mercado laboral, que es condición para la residencia legal y para el acceso a otras prestaciones. Por tanto, el «empleo» desempeña un papel clave en la gobernabilidad de la inmigración a nivel estatal, pues es la regularización del mercado de trabajo uno de los instrumentos con los que cuenta el estado para mantener el control sobre la adquisición de la nacionalidad (ciudadanía).

8. Un ejemplo lo tenemos en los nuevos estatutos de autonomía de Cataluña y de Andalucía.

9. Se aconseja la lectura del capítulo del análisis de la legislación y de los anexos metodológicos para mejor comprensión de esta parte (Iglesias de Ussel et al., 2010). 
En el marco del debate entre transnacionalismo y nacionalismo metodológico, hay una lucha entre quienes piensan que las actividades transnacionales erosionan la soberanía de los estados nacionales (Soysal, 1994; Waldinger y Fitzgerald, 2004), lo cual hace peligrar su existencia, y entre quienes no ven esa oposición excluyente entre ambas perspectivas y consideran que los estados nacionales seguirán durante mucho tiempo manteniendo su soberanía e incluso reforzándola frente a esas posibles amenazas, puesto que su papel no se queda (desde una visión crítica) en la simple homogeneización de la sociedad (Ibáñez Angulo, 2008). En términos usados anteriormente, el debate entre transnacionalismo y nacionalismo metodológico se halla entre la vía de razonamiento conservadora y revolucionaria cuando se habla del futuro del estado nación.

\subsection{El nivel regional}

El análisis ${ }^{10}$ de la gobernabilidad a nivel de las comunidades autónomas destaca principalmente la responsabilidad de los gobiernos regionales en la integración social de los inmigrantes (Iglesias de Ussel et al., 2010), ya que son las competencias directamente relacionadas con la integración las que están transferidas a las regiones (educación, sanidad, servicios sociales, etc.). ¿Cuál es el papel del empleo en el nivel regional dentro de la gobernabilidad de la inmigración? El «empleo» (área laboral) forma parte del conjunto de medidas que los gobiernos autonómicos promueven para la integración social de los inmigrantes dentro de sus planes de integración. Además, en los últimos años, el «empleo», especialmente la competencia sobre los cupos de extranjeros y todas las cuestiones administrativas relativas a la adquisición de permisos de trabajo, se ha convertido en elemento de disputa entre el Estado y las regiones, ya que en los nuevos estatutos de autonomía, como el de Cataluña, se ha demandado al Estado la transferencia de esta competencia.

¿Qué nos aporta el marco teórico formado por el transnacionalismo y el nacionalismo metodológico a nivel de las regiones? Algunas regiones, como el País Vasco, Cataluña, la Comunidad Valenciana o Andalucía, han desarrollado, en sus planes de inmigración, modelos teóricos de «ciudadanías» alternativas al modelo del «estado nación». Al hacer esto, en parte se sitúan dentro de las aportaciones de la perspectiva transnacional a los nuevos modelos de ciudadanía en contextos de inmigración y pluralismo cultural. Pero, por otra parte, caen también en el nacionalismo metodológico, ya que, en el intento de contrarrestar el poder y el control del estado nación, fortalecen el poder y el control de la «región nación», que, aunque con otro nombre, acaba por tener la misma estructura que el «estado nación». La adquisición de las competencias sobre el mercado de trabajo se convierte en el primer paso para controlar el acceso a los nuevos modelos de ciudadanía de carácter regional y a veces nacionalista, ya

10. En el capítulo 4 del informe (Iglesias de Ussel et al., 2010), se encuentran las conclusiones y los resultados del análisis de los planes de integración de un buen número de comunidades autónomas. 
que son ciudadanías reconocidas dentro del ámbito de la comunidad autónoma. Al final, las comunidades autónomas acaban convirtiéndose en pequeños «cuasi estados», donde los paradigmas del «estado nación» descritos por el nacionalismo metodológico siguen estando vigentes.

Por último, una muestra de los efectos del nacionalismo metodológico en la gobernabilidad de la inmigración a nivel regional es el aumento de los estudios de carácter regional, los cuales identifican la unidad de análisis (sociedad) con los límites administrativos de la región, uniformizan y homogeneizan a toda la población y a la realidad social regional. Se observa que la división de las disciplinas en categorías nacionales que se critica en el nacionalismo metodológico (Wimmer y Schiller, 2002a) vuelve a darse a nivel regional y aparecen escuelas y centros de carácter «regional», los institutos «regionales» de estadística, etc., que expanden y reproducen las limitaciones del nacionalismo metodológico a un nivel de análisis más reducido, pero, en definitiva, con esquemas análogos.

\subsection{El nivel local y provincial}

Sin poder extendernos aquí sobre la metodología de análisis del nivel local y provincial ${ }^{11}$, diremos que, en este nivel, hemos analizado cuantitativamente el mercado laboral de las principales provincias con inmigración, distinguiendo entre la situación de los inmigrantes y la de los nacionales. ¿Cuál era el objetivo? Demostrar que, al analizar la realidad social en un nivel inferior al regional dentro de cada región, podemos hallar una gran heterogeneidad en el mercado de trabajo (nacionales e inmigrantes) y que, por tanto, analizar la inmigración a nivel regional (sin descender a unidades menores) impide observar las diferencias que se dan dentro de una misma región, entre sus provincias y municipios, $y$, especialmente, se impide observar las semejanzas que hay entre provincias y municipios de una región con los de otra región, algo que parece mucho más interesante a la hora de encontrar soluciones y de realizar experiencias comunes para la gestión eficaz de la inmigración.

11. Los resultados que este análisis proporciona no pueden exponerse aquí por su extensión, pero empíricamente se comprueba la similitud de la estructura del mercado de trabajo de provincias que pertenecen a regiones diferentes. Por ejemplo, al fijarnos en la población inmigrante, las provincias de Almería, Murcia y Lleida presentan estructuras muy parecidas, especialmente por ser provincias agrícolas. También destacan las similitudes entre Málaga y las provincias canarias. O se refleja en el caso de Andalucía la diferencia entre Sevilla y Granada o Almería. Encontramos indicadores de la heterogeneidad de la inmigración y del mercado de trabajo en provincias de una misma región y nos preguntamos por el sentido que tienen políticas regionales muy generalistas para realidades sociales muy diversas dentro de su ámbito. Nos preguntamos por qué no existe más coordinación entre las regiones o entre los gobiernos locales para sacar experiencias comunes de aquellos casos que presentan un gran parecido. En definitiva, vemos que el proceso de descentralización del Estado a las comunidades debe continuar hacia abajo, a fin de pasar el mayor número de competencias posibles a los municipios y darles mayor autonomía y responsabilidad para gestionarse. 
Desde el nacionalismo metodológico y el transnacionalismo, la aportación al nivel local pasa, por un lado, por continuar evitando las consecuencias (especialmente homogeneizar la realidad social) de tener al «estado nación» o a la «región nación» como principio organizador de la sociedad. Por otra parte, recientes estudios migratorios desde lo local (Portes, 2009; Portes et al., 2008a; Schiller y Caglar, 2009; Schiller y Caglar, 2007) nos muestran cómo, desde este nivel de análisis, se pueden conocer mejor los efectos de las migraciones transnacionales. En el caso de Portes (2009), se comprueba cómo las migraciones pueden favorecer el desarrollo de los países de origen de los inmigrantes, pero estudiando a nivel local las actividades transnacionales entre la ciudad de origen y la de destino. En el caso de Schiller y Caglar (2009, 2007), el papel de las migraciones en el posicionamiento de las localidades en las jerarquías de poder global es de gran utilidad para conocer el potencial de los municipios y las provincias, no simplemente dentro de España, sino a nivel global, que es donde realmente muchas localidades compiten ${ }^{12}$ hoy día.

\section{Conclusiones}

Los tres pilares que la UE define como pilares de las políticas de inmigración son el control de los flujos, la integración y la cooperación al desarrollo. En el estudio de la gobernabilidad de la inmigración en España a partir del debate entre transnacionalismo y nacionalismo metodológico resulta significativo observar cómo cada uno de esos pilares encuentra su sitio en los tres niveles de gobierno analizados: al Estado le corresponde el control (social), pero que es también control de los flujos, control en el acceso a la ciudadanía clásica, donde vemos las consecuencias del nacionalismo metodológico que ha predominado en la modernidad y donde observamos cómo el acceso al mercado de trabajo es uno de los principales instrumentos con los que cuenta el Estado para limitar el acceso a la ciudadanía. La integración social es el principal objetivo de los gobiernos regionales, algo que les compete a ellos por una mera cuestión de descentralización de competencias desde el Estado. Vemos cómo también el empleo o las competencias sobre el mercado de trabajo han sido objeto de disputas entre los gobiernos regionales y el Estado, porque interpretamos, desde una perspectiva sociológica, que la mayor competencia sobre el mercado de trabajo por parte de las regiones compensa el poder del

12. Un ejemplo de este tipo de análisis sería el papel de la inmigración en dos ciudades como Madrid y Barcelona dentro del posicionamiento estratégico o de poder que cada una ostenta a nivel global. Los estudios entre Madrid y Barcelona para determinar qué ciudad tiene más peso dentro de España deben dar paso a estudios en los que se analice a Madrid y Barcelona, no como dos ciudades que compiten entre ellas y dentro del marco nacional, sino como dos ciudades que buscan situarse en un buen puesto dentro de las jerarquías del poder financiero, industrial, tecnológico, etc., pero a nivel global. Ciudades de menor tamaño también deben empezar a compararse no con ciudades vecinas dentro de la misma región o en el territorio nacional, sino con aquellas ciudades extranjeras que están compitiendo con ellas en la atracción de inversores, de capital, de tecnología, etc. 
Estado sobre el acceso a la ciudadanía clásica. Además, frente a esa ciudadanía clásica, la perspectiva transnacional aporta alternativas ligadas al trabajo y a la residencia (no a la nacionalidad) que las regiones llevan un tiempo barajando, como son la ciudadanía inclusiva, plural, cívica, residente, etc. Por último, en el nivel local (provincial), observamos cómo los más recientes estudios desde la perspectiva transnacional colocan en este nivel las posibilidades de cooperación al desarrollo mediante actividades transnacionales de los inmigrantes, con lo cual colaboran no sólo al desarrollo de sus localidades de origen, sino también al desarrollo y buen posicionamiento de las ciudades de acogida en las jerarquías de poder global.

Por todo esto, consideramos que el actual debate entre nacionalismo metodológico y transnacionalismo, más que aspirar a alcanzar un ganador, debe seguir contribuyendo al análisis de la realidad social con toda su complejidad, y eso significa aceptar que el estado nación sigue siendo, de momento, principio organizador de la mayoría de las instituciones y los organismos de nuestro tiempo y que, simultáneamente, se identifican nuevas circunstancias que el hecho migratorio resalta en algunas regiones del planeta y que tienen que ver con una manera de observar la realidad más flexible y sin limitaciones clásicas como las derivadas por el estado nación.

\section{Referencias bibliográficas}

Aja, Eliseo; Montilla, José A. y Roig, Eduardo (2006). Las comunidades autónomas y la inmigración. Valencia: Tirant lo Blanch.

Aláez Corral, Benito (2006). Nacionalidad, ciudadanía y democracia: ¿A quién pertenece la Constitución? Madrid: Centro de Estudios Políticos y Constitucionales.

Aparicio, Rosa; Tornos, Andrés e Instituto de Migraciones y Servicios SociaLES (2002). El estado de bienestar y la inmigración en España. Madrid: IMSERSO.

Arango, Joaquín; Alonso, Josep y AJA, Eliseo (2008). La inmigración en tiempos de crisis. Barcelona: Bellaterra.

Beck, Ulrich (2007). «The Cosmopolitan Condition: Why Methodological Nationalism Fails». Theory, Culturee'Society, 24 (7-8), 286-290.

- (2008). ¿Qué es la globalización?: Falacias del globalismo, respuestas a la globalización. Barcelona: Paidós.

Blanc, C.S.; BasCh, L. y Schiller, N.G. (1995). «Transnationalism, Nation-States, and Culture». Current Anthropology, 36 (4), 683-686.

Bonino, Carla; Aragón, Jorge; Rocha, Fernando; CC.OO. (Confederación SindiCAL) y Fundación Io De Mayo (2004). Los planes de las Comunidades Autónomas para la integración social de las personas inmigrantes. Madrid: Confederación Sindical de Comisiones Obreras.

Chernilo, Daniel (2006). «Social Theory’s Methodological Nationalism: Myth and Reality». European Journal of Social Theory, 9 (1), 5-22.

FAIsT, Thomas (2000a). «Transnationalization in international migration: Implications for the study of citizenship and culture». Ethnic and Racial Studies, 23 (2), 189-222.

- (2000b). The volume and dynamics of international migration and transnational social spaces. Oxford: Oxford University Press. 
FAIst, Thomas y Özveren, Eyüp (2004). Transnational social spaces: agents, networks and institutions. Ashgate: Burlington, VT.

Gil Araújo, S. (2009). "La gestión de la cuestión nacional: España y Cataluña en perspectiva». En: Zapata-Barrero, R. (ed.). Políticas y gobernabilidad de la inmigración en España. 1a ed. Barcelona: Ariel.

Glaser, Barney y STrauss, Anselm (1967). The discovery of grounded theory: Strategies for qualitative research. Chicago: Aldine.

Goldring, Luin; Krishnamurti, Sailaja y Ebrary InC (2007). «Organizing the transnational labour, politics, and social change». Vancouver: UBC Press.

Gowricharn, Ruben (2009). "Changing forms of transnationalism». Ethnic and Racial Studies, 32 (9), 1619-1638.

Guarnizo, Luis Eduardo; Portes, Alejandro y Haller, William (2003). «Assimilation and Transnationalism: Determinants of Transnational Political Action among Contemporary Migrants». American Journal of Sociology, 108 (6), 1211-1248.

Hammar, Tomas (1990). Democracy and the nation state: Aliens, denizens and citizens in a world of international migration. Aldershot: Avebury.

Hix, Simon y Noury, Abdul (2007). «Politics, Not Economic Interests: Determinants of Migration Policies in the European Union». International Migration Review, 41 (1), 182-205.

IbÁÑez Angulo, Mónica (2008). «De la identidad nacional a la ciudadanía transnacional: Procesos migratorios y espacios transnacionales». En: Nuevos retos del transnacionalismo en el estudio de las migraciones. Barcelona. [http://docsgedime. files.wordpress.com/2008/02/monicaibanez.pdf, consulta: 20 de marzo de 2010]

Iglesias de Ussel, Julio; Trinidad, Antonio; Soriano, Rosa; Cantón, Javier y Morcillo, Álvaro (2010). Las politicas de integración social de los inmigrantes en las Comunidades Autónomas españolas: desarrollo y evaluación. Bilbao: Fundación BBVA.

Kivisto, Peter (2001). "Theorizing transnational immigration: a critical review of current efforts». Ethnic and Racial Studies, 24 (4), 549-577.

LevitT, Peggy (2001). The transnational villagers. Berkeley: University of California Press.

Lucas, Javier de y Díez Bueso, Laura (2006). La integración de los inmigrantes. Madrid: Centro de Estudios Políticos y Constitucionales.

Lucas Martín, Javier de (2008). Los derechos de participación como elemento de integración de los inmigrantes. $1^{\text {a }}$ ed. Madrid: Fundación BBVA.

LlOPIS GoIG, Ramón (2007). «El "nacionalismo metodológico” como obstáculo en la investigación sociológica sobre migraciones internacionales». Empiria: Revista de Metodología de Ciencias Sociales, 13, 101-120.

Martins, Herminio (1974). "Time and Theory in Sociology». En: Rex, J. (ed.). Approaches to sociology: An introduction to major trends in British sociology. Londres: International Library of Sociology.

Merton, Robert (1968). Social theory and social structure. 1968 enl. Edition. Nueva York.

Miret i Serra, A. (2009). "La gestión de la división de competencias en materia de inmigración». En: Zapata-Barrero, R. (ed.). Politicas y gobernabilidad de la inmigración en España. $1^{\mathrm{a}} \mathrm{ed}$. Barcelona: Ariel.

Parsons, Talcott (1999). El sistema social. $1^{\text {a }}$ ed. Madrid: Alianza.

Pérez-Díaz, Víctor; Álvarez-Miranda, Berta y González-Enríquez, Carmen (2001). España ante la inmigración. Barcelona: Fundación La Caixa. 
Portes, A.; Escobar, C. y Arana, R. (2008a). «Bridging the gap: transnational and ethnic organizations in the political incorporation of immigrants in the United States.. Ethnic and Racial Studies, 31 (6), 1056-1090.

Portes, Alejandro; Guarnizo, Luis y Landolt, Patricia (1999). «The study of transnationalism: Pitfalls and promise of an emergent research field». Ethnic and Racial Studies, 22 (2), 217-237.

- (2008b). La globalización desde abajo: transnacionalismo inmigrante y desarrollo: La experiencia de Estados Unidos y América Latina. $1^{\text {a }}$ ed. Buenos Aires: Paidós.

PORTES, Alejandro (1999). "Conclusion: Towards a new world: The origins and effects of transnational activities». Ethnic and Racial Studies, 22 (2), 463-477.

- (2001). «Introduction: the debates and significance of immigrant transnationalism». Global Networks: A Journal of Transnational Affairs, 1 (3), 181-194.

- (2009). "Migration and development: reconciling opposite views». Ethnic and Racial Studies, 32 (1), 5-22.

RÉE, J. (1992). «Internationality». Radical Philosophy, 60, 3-17.

SASSEN, Saskia (1996). Losing control?: Sovereignty in an age of globalization. Nueva York: Columbia University Press.

Schiller, N.G. (1999). "Transmigrants and Nation-States: Something Old and Something New in U.S. Immigrant Experience». En: Hirschman, C.; Dewind, J. y Kasinitz, P. (eds.). The handbook of international migration: The American experience. Nueva York: Russell Sage Foundation.

Schiller, N.G.; Basch, L. y Blancszanton, C. (1992a). "Towards a Definition of Transnationalism: Introductory-Remarks and Research Questions». Annals of the New York Academy of Sciences, 645, R9-R14.

- (1992b). "Transnationalism: A New Analytic Framework for Understanding Migration». Annals of the New York Academy of Sciences, 645, 1-24.

Schiller, N.G.; Caglar, A. y Guldbrandsen, T. C. (2006). «Beyond the ethnic lens: Locality, globality, and born-again incorporation». American Ethnologist, 33 (4), 612-633.

Schiller, Nina Glick y Caglar, Ayse (2007). Migrant incorporation and city scale: Towards a theory of locality in migration studies. Malmö: Malmö Institute for Studies of Migration, Diversity and Welfare (MIM). Department of International Migration and Ethnic Relations (IMER).

- (2009). "Towards a Comparative Theory of Locality in Migration Studies: Migrant Incorporation and City Scale». Journal of Ethnic and Migration Studies, 35 (2), 177-202.

Smith, Anthony D. (1979). Nationalism in the Twentieth Century. Oxford: Martin Robertson.

Smith, Michael y Guarnizo, Luis (1998). Transnationalism from below. New Brunswick, N.J.: Transaction Publishers.

Solé, Carlota; Izquierdo, Antonio y Alarcón, Amador (2005). Integraciones diferenciadas: Migraciones en Cataluña, Galicia y Andalucía. Barcelona: Anthropos, Editorial del Hombre.

Soysal, Y.N.G. (1994). Limits of citizenship: Migrants and postnational membership in Europe. Chicago, Ill.: University of Chicago.

Vertovec, S. (1999). "Conceiving and researching transnationalism». Ethnic and Racial Studies, 22 (2), 447-462.

Waldinger, Roger y Fitzgerald, David (2004). «Transnationalism in Question». American Journal of Sociology, 109 (5), 1177-1195. 
Wimmer, A. y Schiller, N.G. (2002a). «Methodological nationalism and beyond: nation-state building, migration and the social sciences». Global Networks: A Journal of Transnational Affairs, 2 (4), 301-334.

- (2002b). «Methodological nationalism and the study of migration». Archives Europeennes de Sociologie, 43 (2), 217-240.

- (2003). «Methodological nationalism, the social sciences, and the study of migration: An essay in historical epistemology». International Migration Review, 37 (3).

Zapata-BARrero, Ricard (2003). "La ciudadanía en contextos de multiculturalidad: Procesos de cambios de paradigmas». Anales de la Cátedra Francisco Suárez, 37, 173-199.

- (2005). "Una nueva "filosofía” de la UE: Tradición versus innovación en la propuesta de Ciudadanía Cívica». En: II Seminario Inmigración y Europa. Barcelona: CIDOB.

- (2009a). Fundamentos de los discursos politicos en torno a la inmigración. Madrid: Trotta.

- (2009b). Políticas y gobernabilidad de la inmigración en España. 1a ed. Barcelona: Ariel.

Zapata-Barrero, Ricard y Pinyol, Gemma (2008). Los gestores del proceso de inmigración: Actores y redes de actores en España y Europa. Barcelona: Fundación CIDOB.

Zapata-Barrero, Ricard y Requejo, F. (2001). Ciudadanía, democracia y pluralismo cultural: Hacia un nuevo contrato social. Rubí (Barcelona): Anthropos. 